\title{
Exploring Innovative and Challenging Applications of EBSD in the Geological and Biological Sciences
}

P.W. Trimby, ${ }^{*}$ and S.C. Piazolo**

* The Australian Centre for Microscopy \& Microanalysis, The University of Sydney, NSW 2006, Australia

** The Department of Earth and Planetary Sciences, Macquarie University, Sydney, NSW 2109, Australia

Introduction: Since its commercialization in the early 1990s, electron backscatter diffraction (EBSD) has become a routine characterization technique in the geological sciences. Despite the fact that the majority of rock-forming minerals are insulating, have relatively low symmetry and produce weak diffraction patterns, modern EBSD systems are able to analyze rock microstructures with a high degree of reliability and at relatively high speeds. The majority of geological applications are in the field of structural geology, notably for the measurement of microtexture (lattice preferred orientation) in deformed rock samples. In recent years, there have been increasing applications of EBSD into more diverse fields of the geosciences, such as into palaeontology [1], glaciology [2, 3] and planetary science [4]. Additionally, EBSD is starting to make an impact in the field of biology and biomaterials [5]. In this paper we present some recent challenging and innovative applications of the EBSD technique in both the geological and biological sciences, focusing on specific material challenges and explaining the approaches used to solve particular microstructural problems.

Highly deformed rock samples. The deformation of rocks usually occurs at relatively high temperatures, resulting in ongoing recovery of the microstructure and limiting the dislocation density. This is known as dynamic recrystallization in the geological literature, and is analogous to hot-rolling techniques in metallurgy. The resulting microstructures, although challenging, can be successfully analyzed using automated EBSD. An example orientation map from a highly deformed quartz mylonite is shown in figure 1. The relatively small spacing between the analysis points (500 $\mathrm{nm}$ ) can cause charging and specimen damage issues, but is offset by the high analysis speed of approximately 100 points / second, routinely achievable with modern EBSD systems. More highly deformed samples, such as those founded in shocked minerals (e.g. as a result of meteorite impacts) or in earthquake zones (e.g. pseudotachylites) can pose significant challenges for EBSD analysis. In such cases, optimizing spatial resolution (e.g. by lowering the beam energy) and increasing the speed of the analysis assist in the acquisition of high quality data.

Biomineralized structures. Many shell structures can be measured successfully using EBSD. Many such structures are made of the mineral calcite $\left(\mathrm{CaCO}_{3}\right)$, a relatively easy mineral to analyze, although sample preparation can be critical in samples with significant inter-crystalline protein or with high porosity. Aragonite, a meta-stable polymorph of calcite, deteriorates rapidly when exposed to an electron beam and is therefore more difficult to analyze using the EBSD technique. In many cases it is necessary to sacrifice the quality of the diffraction patterns in order to maximize the analysis speed, thereby reducing the effect of beam damage. The orientation map in figure 2 was collected from a section through a common sea snail (Angaria delphinus): the clear layers of aragonite are typically only 1-2 $\mu \mathrm{m}$ in width, necessitating a relatively small step size $(100 \mathrm{~nm})$. Some filtering of the raw data was necessary in order to produce the final orientation map, but such an analysis permits a thorough investigation of the relationship between crystal orientation and 
biomineralization. Increasingly EBSD is also being used to characterize fossilized structures, including trilobite eyes [6] and dinosaur eggshells [7].

Ice. Naturally the measurement of ice samples using EBSD poses particular challenges, particularly for sample preparation. Diffraction patterns from ice grains are relatively high quality (fig. 3), but surface sublimation or frost effects due to an incorrect balance between the sample temperature and the chamber vacuum level can result in rapid degradation of the analysis surface, rendering EBSD measurements impossible. Despite these challenges, EBSD measurements of ice samples are providing significant insights into the deformation processes associated with ice flow.

It is certain that EBSD will continue to be used as a tool for analyzing an ever-increasing range of crystalline materials and that further advances in sample preparation techniques as well as developments in both EBSD hardware and software will further promote these diverse applications.

\section{References}

[1] A. Pérez-Huerta et al., Palaeontology 50 (2007), 757-763.

[2] D. Iliescu et al., Microsc. Res. Technol. 63 (2004), 183-187.

[3] S. Piazolo et al., J. Microsc. 230 (2008), 509-519.

[4] M. Zolensky and T. Mikouchi, Microsc. Microanal. 16 (Suppl 2) (2010), 676-677

[5] P. Dalbeck and M. Cusack, Crystal Growth and Design, 6 (2006), 2558-2562.

[6] M. R. Lee et al., Palaeontology 50 (2007), 1031-1037.

[8] G. Grellet-Tinner et al., Gondwana Research (in press)

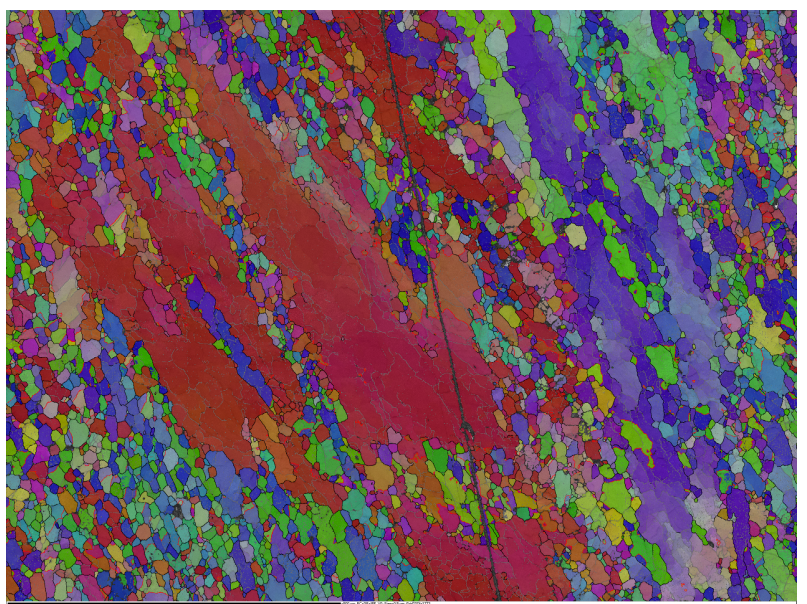

FIG. 1. Orientation map of a highly deformed quartz rock. The map consists of $\sim 4.2$ million analysis points at $500 \mathrm{~nm}$ spacing. The scale bar marks $0.5 \mathrm{~mm}$.

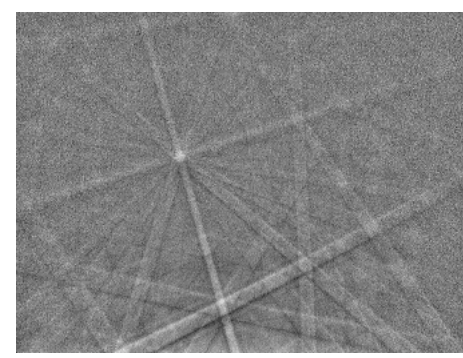

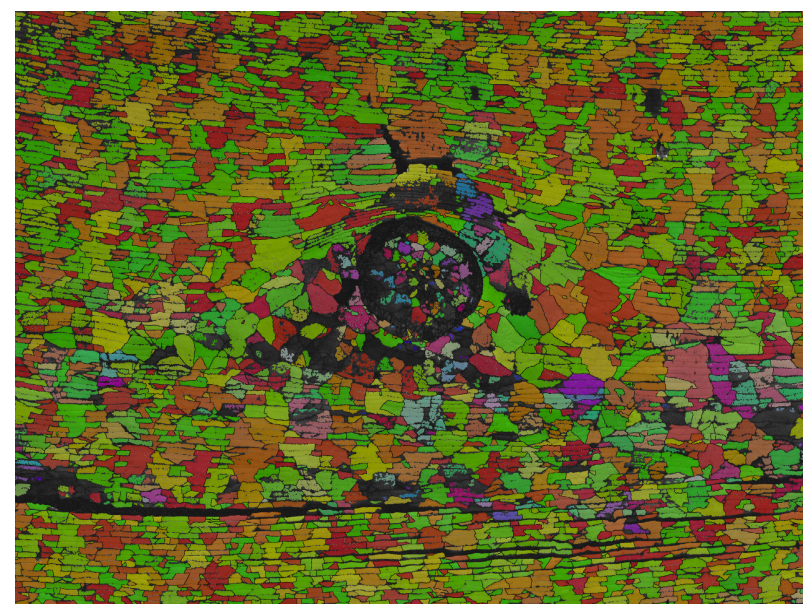

FIG. 2. Orientation map of a cross section through the shell of a common sea snail. The map consists of $\sim 4.2$ million analysis points, at $100 \mathrm{~nm}$ spacing. The scale bar marks $100 \mu \mathrm{m}$.

FIG. 3. Example electron backscatter diffraction pattern from an ice crystal. 